\title{
Lieux touristiques et gestion de l'altérité
}

Réflexions autour des pratiques de sociabilité

\section{Vincent Coëffé}

\section{(2) OpenEdition}

\section{Journals}

Édition électronique

URL : http://journals.openedition.org/tourisme/76

DOI : $10.4000 /$ tourisme.76

ISSN : 2492-7503

Éditeur

Éditions touristiques européennes

\section{Édition imprimée}

Date de publication : 1 décembre 2013

Pagination : 11-18

ISSN : 2109-5671

Référence électronique

Vincent Coëffé, «Lieux touristiques et gestion de l'altérité », Mondes du Tourisme [En ligne], 8| 2013, mis en ligne le 01 juin 2015, consulté le 19 avril 2019. URL : http://journals.openedition.org/ tourisme/76 ; DOI : 10.4000/tourisme.76

\section{$@ \Theta \Theta \Theta$}

Mondes du tourisme est mis à disposition selon les termes de la licence Creative Commons Attribution - Pas d'Utilisation Commerciale - Pas de Modification 4.0 International. 


\section{Lieux touristiques et gestion de l'altérité Réflexions autour des pratiques de sociabilité}

\section{VINCENT COËFFÉ}

[vincent.coeffe@univ-angers.fr]

Maître de conférences en géographie,

UFR Ingénierie du tourisme, du bâtiment, et des services (UMR CNRS ESO 6590), Angers

Résumé. Le tourisme est un opérateur puissant de la mondialisation qui pose la question de la relation des touristes à l'altérité. Mais alors que la sociabilité constitue un ingrédient majeur des pratiques touristiques, son articulation à la dimension spatiale, notamment à travers les lieux, est rarement appréhendée. C'est l'objectif de cet article que de lancer une analyse permettant de mettre au jour au moins trois configurations: l'entre-soi, l'immersion métropolitaine, le modèle cruséen.

Abstract. Tourism is a powerful operator of the globalization, which asks the question of the tourists' relation to the otherness. But while the sociability constitutes a major ingredient of the tourist practices, its articulation with the spatial dimension, in particular through places, is rarely explored. The aim of this article is to start an analysis allowing to enlighten at least three configurations: "being with the same", the metropolitan immersion, the model of "Robinson Crusoé". 
$\mathbf{L}$ e tourisme, invention britannique de la seconde mondialisation, peut être appréhendé comme un puissant moteur de mise en contact des lieux du monde, même si les échelles concernées par les mobilités touristiques ne sont pas forcément calées sur la plus grande extension. Les premières destinations mondiales sont pour l'essentiel européennes et les échanges entre elles continuent intensément. Ainsi, même s'il faut relativiser les statistiques produites par l'OMT (Organisation mondiale du tourisme), qui intègre notamment dans le tourisme des mobilités qui ne s'inscrivent pas dans la recréation (ensemble des pratiques rompant avec le quotidien, y compris avec la sphère professionnelle), les ordres de grandeur montrent que "sur le continent européen, $87 \%$ des séjours internationaux sont le fait de ressortissants européens" (Dehoorne, Saffache et Tatar, 2008).

Mais contrairement à ce qui est ressassé dans le discours commun comme dans la vulgate scientifique (Christin, 2008), le monde n'a pas été tout à fait "uniformisé", voire "banalisé" par le tourisme même si cette forme de mobilité a rendu (et rend) possible la connaissance de lieux autres qui ont gagné en proximité. Par le seul fait de se déplacer temporairement vers des lieux du hors-quotidien, les touristes valorisent un différentiel d'altérité (la qualité de ce qui est autre) sans lequel ils n'auraient pas de raison de retrouver ailleurs ce dont ils pourraient se saisir $i c i$.
Le constat d'une homogénéisation de l'espace touristique s'appuie bien souvent sur l'observation de ces flux qui n'ont pas cessé d'augmenter à l'échelle du monde depuis les années 1950 notamment, et par la saisie des indices les plus visibles d'une standardisation des lieux pratiqués par les touristes (chaînes hôtelières mondialisées notamment).

Il ne s'agit pas ici de nier certaines logiques de convergence des pratiques, images, valeurs, normes à travers le monde, processus auquel contribuent les acteurs du tourisme. L'aménagement de ce qui est représenté comme "autre" par les touristes potentiels est même une des conditions de leur mobilité, mais selon des modalités qui peuvent considérablement varier d'un individu à l'autre, notamment en fonction de leurs “compétences spatiales", mesurée surtout à travers l'expérience capitalisée des lieux du monde.

Car il s'agit bien ici d'appréhender l'altérité comme un problème et non comme une évidence qui n'appellerait que des jugements de valeur coincés entre idéalisation "du" touriste des "confins" (lieux d'une altérité dite "radicale") et haine du touriste des lieux "dédiés" (lieux du tourisme de masse, offrant les standards attendus).

Nous nous intéresserons ici plus particulièrement à l'altérité sous l'angle de la relation aux autres, que ces derniers soient touristes (cette catégorie posant d'ailleurs problème au sens où il n'existe pas a priori d'identité entre les individus identifiés comme tel) ou "résidents". La socia- bilité est en effet au fondement des pratiques touristiques et en constitue toujours un des ressorts majeurs (Équipe Mit, 20I I), sans que le phénomène ait rencontré jusqu'à présent un intérêt marqué de la part des chercheurs. Certes, des travaux sont disponibles (Amirou, 1995) mais ils ne prennent pas toujours suffisamment au sérieux la dimension spatiale des sociabilités.

Or les lieux touristiques ne sont pas ici de simples cadres des relations sociales mais incarnent en même temps qu'ils rendent possibles les différentes manières de produire des sociabilités. Nous faisons donc l'hypothèse que si les relations sociales en situation touristique prennent place dans le contexte du hors-quotidien et du déplacement à des fins de recréation (reconstitution du corps et de l'esprit, rupture avec la routine), leurs configurations sont plus ou moins changeantes dans le temps, de même que les acteurs du tourisme ont inventé ou maintenu des formes de lieu autorisant (ou non) leur mise en œuvre.

\section{“ENTRE-SOI”, “ENTRE-LIEU”}

Alors que la rencontre est un moteur essentiel des pratiques touristiques, au point d'apparaître souvent comme un impératif moral (le touriste se doit notamment de rencontrer la "vraie" société, celle qui est perçue comme "locale"), celleci met sans doute d'abord en relation des touristes avec d'autres touristes.

Cela est vrai de l'aristocratie britannique qui crée au XVIII ${ }^{\mathrm{e}}$ siècle les 
premiers lieux touristiques en recherchant de nouvelles formes de distinction. Il s'agit d'y maintenir les logiques d'intenses sociabilités circulant à l'intérieur de cette classe sociale. L'espace privé des châteaux et hôtels reste alors la "technologie spatiale" privilégiée pour assurer la reproduction sociale par l'entre soi.

Mais c'est vrai aussi lorsque la bourgeoisie s'invite un peu plus tard dans l'univers du tourisme : il s'agit là encore de garantir le fonctionnement des sociabilités mondaines. Encore que l'innovation ne soit pas mince ici. Á Bath, matrice de tous les lieux touristiques (Équipe Mit, 2005), l'élite marchande et financière crée les assembly rooms, lieux du jeu, de la danse et de la boisson à travers lesquels la haute société s'affiche cette fois sur la place "semipublique" (on y est normalement accepté à condition d'être coopté). Des bals y sont organisés qui constituent autant d'occasions pour les jeunes gens à marier de faire leur entrée dans le monde, dans un cadre moins contraignant, plus ouvert (Équipe Mit, 20l I).

Certes la bourgeoisie souhaite entretenir ses mondanités dans les assembly rooms qui sont aussi des lieux autorisant la mise en scène d'une nouvelle étiquette. Mais la haute société ne peut empêcher totalement la classe laborieuse d'y accéder, les assembly rooms étant devenus l'objet de toutes sortes de convoitise et le lieu où les choses se passent (Équipe Mit, 2005).

Ainsi, alors que les stations créées par et pour les premiers touristes ont été conçues comme des lieux de l'entre-soi, elles n'étaient pas agencées pour autant comme de véritables enclaves hermétiques et la haute société a dû très tôt composer avec une certaine mixité sociale, d'autant que la mise en scène de pratiques ostentatoires n'avaient de sens qu'en présence des classes laborieuses comme le montre le déploiement des promenades qui permettaient de parader (Debié, 1993).

Cela dit, l'entre-soi est une figure de la sociabilité touristique qui traverse en réalité toute l'histoire du tourisme. Si les stations ont finalement garanti une certaine porosité en permettant la circulation et l'installation d'acteurs qui n'étaient pas des touristes (mais des résidents "permanents" notamment), un nouveau type de lieu est apparu, capable de garantir encore mieux l'entre-soi touristique à mesure qu'émerge le tourisme "de masse" et la fréquentation toujours plus nombreuse de lieux à fort différentiel d'altérité. Le comptoir, qui a constitué une invention littorale majeure dans le monde du tourisme (Duhamel et Violier, 2009) incarne sous la forme du villageclub ce passage mais il ne s'agit plus de produire forcément un entre-soi "de classe" (oisive). C’est ce que montre un modèle de lieu comme celui du Club Med. Le village-club avait été d'emblée conçu par ses fondateurs (Blitz et Trigano) comme un territoire garantissant l'accessibilité du plus grand nombre. Et la montée en gamme récente du touropérateur à la suite de difficultés financières, ne doit pas occulter le projet initial. En accord avec les principes du tourisme social qui émergent dans les années 1950, le Club Med avaient imaginé une place pour les segments modestes (Équipe Mit, 20I I).

En ouvrant un village-club aux Baléares et en Corse dès ce momentlà, l'agencement en comptoir garantissait aux touristes européens une distance suffisante avec l'environnement immédiat en même temps qu'il permettait de nouvelles proximités avec des mondes inconnus pour beaucoup d'entre eux (Équipe Mit, 2005).

Alors que l'aventure n'était pas forcément avec la société locale, elle était en tout cas privilégiée à l'intérieur du club entre des touristes pour la plupart anonymes les uns pour les autres, mais qui portaient certaines valeurs montantes comme la libération sexuelle. La réputation du Club Med s'est construite en partie sur ce mode de sociabilité qui fait de l'autre touriste un partenaire amoureux potentiel (quand ce n'est pas le "gentil organisateur"). Le voyagiste constitue d'ailleurs encore aujourd'hui l'emblème de ce type de pratiques touristiques, malgré un repositionnement qui cherche aussi à prendre quelque distance avec cet héritage parfois un peu encombrant.

Alors que l'Autre peut avoir plusieurs figures (l'autre touriste que l'on n'a pas l'habitude de rencontrer dans le contexte du quotidien, l'autre résident, etc.), le comptoir qui rend possible l'entre-soi touristique peut très bien constituer une pointe avancée pour se rapprocher de la société 
locale. La force du comptoir, pour l'individu mal doté en “compétences spatiales" notamment, est son organisation éprouvée qui peut se lire aussi à travers les différentes formes de standardisation qu'il est capable d'offrir (hébergement, restauration, etc.), tout en assurant la coprésence rassurante d'un groupe construit autour d'une même position : celle d'être ici, temporairement, dans un contexte de recréation.

De ce point de vue, le comptoir fonctionne comme un "sas", une enveloppe protectrice qui permet d'affronter individuellement et collectivement l'altérité (Équipe Mit, 2002 ; Ceriani, 2005). Cette fonction d' "entre-lieu” (Lapierre, 2006) se retrouve d'ailleurs plus ou moins dans les voyages à forfait qui délestent les touristes d'une certaine dose d'inconnu et qui leur permettent de se rendre davantage disponibles aux autres.

Dans ce cadre, la station reste encore aujourd'hui largement valorisée pour les rencontres possibles qu'elle autorise, notamment entre touristes. Une des formes les plus abouties de ce projet est donnée à voir par la pratique du springbreak (Équipe Mit, 20l I).

Alors que cette "pause de printemps" est une invention étasunienne à travers laquelle les étudiants notamment partent une semaine vers des destinations “tropicales" comme Cancun ou Acapulco pour se recréer par la fête, le springbreak se diffuse en Europe où les étudiants élisent ici aussi leurs lieux, certaines stations balnéaires espagnoles comme Lloret de Mar figurant en bonne place. Pratique plus ou moins sulfureuse, suscitant régulièrement le scandale par les excès qui y sont mis en scène, cette forme de recréation n'en demeure pas moins suffisamment "normée" pour être intégrée dans les offres de certains tour-opérateurs (Playayfiesta, par exemple).

L'entre-soi constitue donc une catégorie plastique, capable de varier les échelles de la sociabilité touristique. Être entre-soi, cela peut prendre une grande diversité de formes. Cela peut consister à entretenir les relations au sein d'un groupe d'amis déjà constitué mais dans lequel les membres souhaitent vivre touristiquement leur sociabilité (cela peut d'ailleurs consister, le temps des vacances, à intégrer de nouveaux membres dans le groupe). Ce peut être la volonté pour un individu ou un groupe de privilégier des proximités nationales dans le hors-quotidien comme dans le cas de certains quartiers touristiques à Palma (Majorque, Baléares) dans lesquelles les enseignes et les prestataires de service communiquent en langue germanique.

Ce peut être encore l'entre-soi du couple qui cherche à resserrer des liens conjugaux effilochés par des vies professionnelles fonctionnant à des rythmes différents, demande qui n'a pas échappé à certains voyagistes comme le Club Med, là encore, lequel propose dans certains de ses villages la garde des enfants en plus des prestations qui dégagent les parents des principales contraintes quotidiennes.
Mais ce peut être aussi l'entre-soi touristique d'un individu seul préparant à travers ce retranchement un futur déplacement au cours duquel il s'exposera davantage à la société locale.

\section{BAINS D’ALTÉRITÉ ET LIENS FAIBLES : UNE IMMERSION DANS L'URBANITÉ MÉTROPOLITAINE}

Si stations et comptoirs peuvent favoriser plus ou moins l'entre-soi, ces types de lieu touristique ne sont paradoxalement pas pour rien dans la capacité d'un nombre toujours croissant de touristes à s'accommoder voire à privilégier des sociabilités moins programmées, plus ouvertes à de forts différentiels d'altérité.

En pointillés, et sans que les touristes en soient toujours pleinement conscients, les stations et comptoirs ont permis et permettent encore de réduire la distance (culturelle, cognitive) avec certains lieux, préparant les individus qui en font l'expérience, à davantage d'autonomie avant et pendant leur futur déplacement. Ces deux modèles de lieu ont favorisé la multiplication et l'extension des mobilités touristiques en sorte qu'elles ont contribué à l'accroissement des savoirs géographiques des touristes sur plusieurs générations, y compris en termes de compétences de sociabilité dans des lieux du horsquotidien.

Nous comprenons mieux pourquoi - alors que les discours antiurbains connaissent par ailleurs une longue carrière - les métropoles remportent un succès sans précédent 
auprès des touristes, qui viennent alimenter par la diversité de leurs provenances ces "concentrés de Monde" que sont les grandes villes. Alors que les touristes peuvent toujours opter, à certains moments au moins du séjour métropolitain, pour un retranchement sur leur hôtel aux standards mondialisés, le choix de la métropole est celui d'un lieu qui continuerait de fonctionner sans eux, les touristes ne faisant qu'ajouter à la diversité ambiante qui caractérise l'urbanité, notamment "européenne" (Lévy, 1997). Ainsi, l'entre-soi touristique n'est pas ici le mode de sociabilité privilégié. Le projet des touristes visitant les métropoles est d'abord d'être immergé dans un bain hautement chargé en altérité.

Cela ne signifie pas forcément que les sociabilités avec les autochtones soient "fortes". Si les touristes sont d'abord des citadins, notamment des grandes villes, ils en ont acquis les manières d'être. Autrement dit, ils sont porteurs d'urbanité chaque fois qu'ils se déplacent, dès lors que l'on accepte avec Park l'idée selon laquelle "la ville est quelque chose de plus qu'une agglomération d'individus et d'équipements collectifs [...] ; c'est également quelque chose de plus qu'une simple constellation d'institutions et d'appareils administratifs [...]. La ville est plutôt un état d'esprit [...]" (Grafmeyer et Joseph, 1990, p. 83). Or les chercheurs de l'École de Chicago, qui se sont intéressés notamment aux modes de sociabilité urbaine l'ont bien montré : "L'accroissement du nombre d'babitants d'une communauté au-delà de quelques centaines oblige à limiter la capacité de chaque membre à connaître tous les autres personnellement" (Wirth dans Grafmeyer et Joseph, 1990, p. 266). Dit autrement, le contexte urbain, "établissement relativement important, dense et permanent d'individus socialement hétérogènes" (ibid., p. 262), informe la dimension idéelle de ses habitants, notamment ceux des métropoles qui ont tendance à développer certaines stratégies cognitives: "Si l'incessante mise en contact superficielle d'une quantité de personnes dans la grande ville devait se recouper avec un nombre égal de réactions intérieures, comme dans la petite localité où chacun connaît presque tous ceux qu'il rencontre et entretient avec chacun d'eux une relation effective, on aurait une vie intérieure complètement atomisée et l'on tomberait dans un état mental inconcevable" (Simmel cité par Wirth, ibid., p. 267).

D'où le principe selon lequel "les contacts en ville peuvent effectivement être de face à face, mais ils n'en sont pas moins superficiels, éphémères et segmentaires. La réserve, l'indifférence et l'attitude blasée que les citadins manifestent dans leurs relations peuvent ainsi être considérées comme des dispositifs d'immunisation contre les revendications personnelles et les attentes de la part des autres" (Wirth, ibid).

En visitant une métropole et les virtualités qu'elle offre, les touristescitadins n'adoptent pas forcément une posture différente. Ils y mobilisent au contraire leurs compétences citadines au travers desquelles ils peuvent prendre plaisir à être immergés dans la diversité et la densité propriétés urbaines selon la définition de l'urbanité proposée par Jacques Lévy (1994) -, tout en maintenant le jeu tendu entre proximité et distance aux autres.

Si certains touristes peuvent se délecter du bain de foule en participant à l'animation métropolitaine (qu'ils tendent donc à intensifier), leurs relations aux autres se calent en grande partie sur une gestion des liens faibles (Coëffé, 2009; 2010 ; Équipe Mit, 20I I). Certes la rencontre approfondie n'est pas exclue, et les métropoles touristiques rendent possibles des relations cosmopolites qui peuvent déborder le temps des vacances. Mais le mode de sociabilité le plus ordinaire est celui de la coprésence, régime par lequel la proximité physique n'implique pas forcément une "attention focalisée" (Goffman, 1973) des individus les uns pour les autres, mais davantage une attention diffuse, préservant les "territoires du moi” (ibid.), autrement dit l'espace intime de chacun, que ce dernier soit touriste ou résident. Á rebours des discours convenus sur la superficialité des relations sociales en contexte urbain, Simmel et Wirth ont appréhendé les liens faibles comme un mode positif de sociabilité (in Grafmeyer et Joseph, 1990). Richard Sennett a même montré plus récemment le régime productif opéré par la tension entre proximité et distance dans l'espace public: "Plus les gens ont de barrières tangibles entre eux, plus ils sont sociables, de même qu'ils 
ont besoin d'endroits publics spécifiques dont la seule fonction soit de les rassembler. (...) Les êtres humains ont besoin de se trouver protégés des autres pour être sociables. Augmentez le contact intime, vous diminuez la sociabilité" (1979, p. 24).

Pour autant, nous n'oublierons pas que la pratique touristique ouvre un régime de recréation c'est-à-dire de "relâchement des contraintes", de rupture relative avec un fort autocontrôle des émotions (Stock, 2003), précisément celui qui imprègne ordinairement le style spatial (par la gestion de la distance aux autres) des citadins dans l'espace public. Or si la drague par exemple est un mode de sociabilité qui peut venir rompre "l'inattention polie" (Goffman, 1973) dans l'espace public, il n'est pas rare que cette pratique constitue pour certains touristes un enjeu important de leur relation aux autres, jusqu'à mettre à l'épreuve les normes citadines.

Il faut dire aussi que ce sont ces micro-événements qui donnent de l'épaisseur à l'espace public, et qui peuvent parfois faire bifurquer un itinéraire biographique. L'immersion des touristes dans la foule anonyme des métropoles doit beaucoup à ces petites ruptures dans l'ordinaire, $y$ compris sous la forme des malentendus qui servent aussi à amener à la conscience les normes. La déambulation dans la rue métropolitaine n’a bien souvent pas d'autres motifs que de prendre plaisir à être avec les autres, baigné par le flux différencié des passants dont la flânerie est scandée ici ou là par une interaction minuscule (Coëffé, 20।0).
Ce régime peut d'ailleurs culminer la nuit, l'imaginaire nocturne ayant tendance à voir le contrôle social et les contraintes de rôles se relâcher durant ce temps social (Deleuil, 1994). À travers l'expérience touristique, il est à la fois différent du soi diurne et du soi quotidien. Plus attentif à soi dans le cadre d'un temps non contraint, l'individu temporairement touriste est aussi sans doute plus attentif aux autres, les forts différentiels d'altérité sollicitant plus que jamais l'appareillage sensoriel.

L'espace public métropolitain (mais aussi celui des stations) devient le contexte et l'enjeu d'une exposition aux autres à travers laquelle les jeux de miroir fonctionnent à plein régime. D'où ce travail attentif sur les apparences qui offrent des prises pour les autres passants, dans le contexte d'un anonymat assumé mais dont les membres coprésents cherchent en même temps à ouvrir la "boîte noire" (Coëffé, 2003).

La fréquentation des lieux les plus denses et les plus animés des métropoles, par les touristes, n'est pas chose nouvelle. Elle existe depuis les débuts du tourisme, les plus grandes villes d'Europe ayant constitué les matrices et les réceptacles du phénomène lui-même. Mais la tendance s'est exacerbée dans les dernières années à la faveur de la redistribution des citadins dans l'espace urbain.

Alors que ces derniers habitent de moins en moins les ville-centre durant leur quotidien, au profit des espaces péri voire suburbains, les citadins investissent plus que jamais, durant le hors-quotidien, les lieux aux gradients d'urbanité les plus élevés (où le couple densité-diversité est donc maximisé) (Knafou, 2007).

Cela se traduit notamment par la volonté des touristes de participer aux grands événements métropolitains (et aux micro-événements qui lui sont liés) au cours desquels ils se mêlent aux résidents, comme lors de l'opération "Paris-Plage" par exemple. Les touristes contribuent même à faire de la métropole en général et de la métropole européenne en particulier un événement permanent, en prolongeant l'animation des rues jusqu'aux confins de la nuit (Gravari-Barbas, 2007), et en propulsant certaines villes au rang de "lieu du moment" qui est peutêtre en train de devenir un "moment de lieu", comme c'est le cas de Barcelone et de ses ramblas très fréquentées, aux provenances mêlées (Équipe Mit, 2005).

Le goût de la grande ville peut se lire dans la pratique actualisée du citybreak, une pratique à travers laquelle les individus cherchent à habiter touristiquement une grande ville le temps d'un court séjour.

Certains opérateurs ont saisi là une nouvelle opportunité pour positionner leur offre. Ainsi de Air Berlin qui tire parti du choix des étudiants allemands de passer la nuit du jeudi au vendredi à Palma, tout en les affranchissant de la nécessité de solliciter un hébergement grâce à son réseau dense de lignes activant un large faisceau de villes en Allemagne mais aussi de plus en plus ailleurs en Europe ${ }^{(1)}$. 


\section{LE MODÈLE CRUSÉEN : SEUL AVEC LE GOÛT DES AUTRES}

Il est toujours possible d'être seul au milieu d'une foule, la proximité des multitudes ne garantissant pas forcément l'investissement collectif de l'individu. Mais outre qu'il est difficile d'être totalement indifférent au contexte d'action, les touristes ont toujours le choix de se tenir à distance des foules si leur projet est de prendre congé du monde.

Ce projet n'est pas scandaleux en soi. Pour autant, un lieu comme la plage crée régulièrement le scandale aussi parce que le régime de sociabilité y laisse de la place pour la distanciation temporaire des individus à l'égard des autres.

C'est à travers la pratique de la sieste que ce retour sur soi s'observe sans doute le mieux. Alors que celleci parcourt un continuum de la somnolence au sommeil, Thierry Paquot a bien montré comment la sieste activait le rapatriement d'un temps à soi, "un moment, plus ou moins long, de mise-en-présence-avec-soi par l'absence, momentanée, d'avec le monde" (Paquot, 1998, p. 55). Il s'agirait d'un moment permettant "la réunion, la réunification, la reconstitution provisoire de notre personnalité éclatée, divisée, éparpillée" (ibid.). Cette discontinuité temporelle et spatiale garantirait ainsi la possibilité de "faire le point" (ibid.) et constituerait un "opérateur d'apaisement du stress, favorisant du même coup la confiance dans le monde" (Coëffé, 2003, p. 667). Autrement dit, le retrait momentané du monde par la sieste, disponibilité à soi-même, peut aussi fonctionner comme un lieu préparant à la disponibilité aux autres. L'immobilité temporaire qui lui est associée, et qui est autorisée par certains lieux comme la plage, peut alors être une manière de rompre avec un quotidien organisé autour d'un régime d'hypermobilité qui oblige à multiplier les rencontres sociales dans le cadre de l'activité professionnelle.

Plus globalement, la volonté de solitude reste pour l'essentiel une parenthèse dans le séjour touristique. Partir seul s'apparente le plus souvent à une volonté d'agrégation aux autres, quoiqu'en disent certains touristes souhaitant par là faire surtout œuvre de distinction. Les professionnels ne s'y sont pas trompés qui proposent depuis plusieurs années des séjours "célibataire" qui affichent en creux une offre de rencontre amicale et/ou amoureuse.

Même quand les touristes décident de partir seuls, en s'affranchissant des encadrements proposés par les opérateurs touristiques, c'est dans le secret espoir de rencontre ou au moins d'accumulation d'un "capital symbolique" quand le séjour en solitaire pourra être échangé au retour à travers l'expérience rapportée (photos, récits, etc.).

La robinsonnade qui fait en même temps florès dans la communication touristique offre une lecture complexe de la relation des individustouristes au monde. Une des interprétations les plus courantes se rapproche de celle de Rousseau, avec les figures de la sédentarité et de l'isolement qui lui sont associées (Urbain, 2002).

Or le modèle cruséen montre tout autre chose, Robinson pratiquant sur son île la double résidence et ne pouvant vivre dans la totale solitude, même si ses relations sociales sont par ailleurs calées sur le système aristocratique (dissymétrie des relations sociales notamment avec la figure de Vendredi). Certes la robinsonnade touristique incarnée par le modèle de l'île-hôtel (Équipe Mit, 2005) permet l'entre-soi de classe. Pour autant, il n'autorise pas seulement la solitude, et le choix du retranchement peut aussi s'inscrire dans une stratégie de distinction de la part de la haute société qui souhaite surtout se tenir à distance de la diversité sociale pour entretenir ses sociabilités mondaines.

Il faut dire aussi que partir seul est une option qui n'est pas partagée par toutes les sociétés, notamment les plus holistiques d'entre elles, où les logiques communautaires l'emportent sur l'individu qui n'est pas, comme dans les sociétés individualistes (lesquelles se sont largement construites en Europe et ses projections, c'est-à-dire aux États-Unis notamment), positionné sur le "même plan que les collectifs et les organisations, acteur à part entière de l'action dans la société" (Durand, Lévy, Retaillé, 1992, p. 24). Pour autant, l'individualisation est un processus désormais mondialisé. Le paradigme nippon est intéressant de ce point de vue, surtout que le tourisme semble y catalyser les changements sociétaux en cours. Alors que les Japonais affrontant de forts 
différentiels culturels, se déplaçaient de manière quasi exclusive en groupe il y encore quelques décennies, il est de moins en moins rare de voir des touristes en provenance du Japon pratiquer seuls certaines destinations européennes ou étasuniennes par exemple, notamment les métropoles.
Cela est autant le signe d'une autonomisation des Japonais à l'égard des opérateurs touristiques qu'une autonomisation croissante des individus au sein de la société nipponne.

Ainsi, alors que la solitude reste très faiblement valorisée par les touristes d'une manière générale, les pratiques touristiques solitaires peuvent très bien signifier la distanciation à l'égard d'un groupe devenu étouffant et le rapprochement vers un Autre plus ou moins désiré.

(I) Nous remercions Miguel Segui Llinas pour nous avoir foumi cette information.

\section{RÉFÉRENCES BIBLIOGRAPHIQUES}

Rachid AmIROu, Imaginaire touristique et sociabilités du voyage, Puf, 1995.

Giorgia Ceriani, Philippe Duhamel, Rémy Knafou et Mathis

STOCK, "Le tourisme et la rencontre de l'autre. Voyage au pays des idées recues", L'Autre. Clinique, cultures et sociétés, vol. 6, 2005. Rodolphe CHRISTIN, Manuel de l'antitourisme, éditions Yago, 2008. Vincent CoËFFÉ, "Le tourisme, fabrique d'urbanité. Matériaux pour une théorie de l'urbain", Mondes du Tourisme, n², 2010.

Vincent CoËFrÉ, "Du site à la métropole touristique : comment les touristes produisent du jeu avec la ville", dans Jean-Pierre LEMASSON et Philippe VIOLIER (dir.), Destinations et territoires, vol. I : Coprésence à l'œuvre, Presses de l'université du Québec, 2009.

Vincent CoËFré,Touristicité idéale. Hawaii, un parcours utopique, thèse de doctorat en géographie, Université de Rouen, 2003.

Franck DEBIÉ, “Une forme urbaine du premier âge touristique : les promenades littorales", Mappemonde, n I, 1993.

Olivier Dehoorne, Pascal SAfFACHe et Corina TATAR, Le Tourisme international dans le monde : logiques des flux et confins de la touristicité, 2008 [http://etudescaribeennes.revues.org/document882.html]. Jean-Michel DeleuIL, Lyon, la nuit. Lieux, pratiques et images, Presses universitaires de Lyon, "Transversales", 1994.

Philippe Duhamel et Philippe Vıolıer, Tourisme et littoral. Un enjeu du monde, "Tourisme", Belin, 2009.

Marie-Françoise DuRAND, Jacques LÉVY et Denis Retallté, Le Monde, espaces et systèmes, Presses de Sciences Po et Dalloz, 1993. ÉQUIPE MIT, Tourismes 3. La Révolution durable, "Mappemonde", Belin, 2011 .
ÉQUIPE MIT, Tourismes 2. Moments de lieux, "Mappemonde", Belin, 2005.

ÉQuipe Mit, TOURISMES I. LIEUX communs, Belin, "Mappemonde”, 2002.

Erving GoffmaN, La Mise en scène de la vie quotidienne; tome 2 : Les relations en public, "Le sens commun", Éditions de minuit, 1973. Yves GrafmeYer et Isaac JOSEPH, L'École de Chicago. Naissance de l'écologie urbaine, "Champ urbain", Aubier, 1990.

Maria Gravari-Barbas, "Á la conquête du temps urbain :

la ville festive des '24 heures sur 24"', dans Philippe DUHAMEL et Rémy KNAFOU, Mondes urbains du tourisme, "Mappemonde", Belin, 2007.

Rémy KNAFOU, "L'Urbain et le tourisme : une construction laborieuse", dans Philippe DUHAMEL, Rémy KNAFOU, Mondes urbains du tourisme, “Mappemonde”, Belin, 2007.

Nicole LAPIERre, Pensons ailleurs, "Folio Essais", Gallimard, 2006

(I réédition 2004).

Jacques LÉVY, Europe. Une géographie, Hachette, 1997.

Jacques LÉVY, L'Espace légitime. Sur la dimension géographique de la fonction politique, Presses de Science Po, 1994.

Thierry PAQUOT, L'Art de la sieste, Zulma, 1998.

Richard SenNetT, Les Tyrannies de l'intimité, "La couleur des idées", Seuil, 1979.

Mathis Stock (coord.), Le Tourisme. Acteurs, lieux et enjeux, "Belinsup Géographie", Belin, 2003.

Jean-Didier URBAIN, "Du mythe à la manière. Résider selon

Robinson en son île", dans Communications, Manières d'habiter, $n^{\circ} 73$, 2002. 